\title{
On the discovery potential of the lightest MSSM Higgs Boson at the LHC
}

\author{
R. Kinnunen†, S. Lehti†, A. Nikitenko $\$$ and P. Salmi† \\ $\dagger$ Helsinki Institute of Physics, Helsinki, Finland \\ $\ddagger$ Imperial College, University of London, London, UK
}

\begin{abstract}
Production of the lightest MSSM Higgs boson h is studied at the LHC. Isorate contours for the $\mathrm{h} \rightarrow \gamma \gamma$ and $\mathrm{h} \rightarrow \tau^{+} \tau^{-}$decay channels are shown in the $\left(\mathrm{m}_{\mathrm{A}}, \tan \beta\right)$ parameter space. Effects of the SUSY parameters, in particular the stop mixing and stop quark mass, are investigated. Search strategies at the LHC are discussed and the discovery potential is calculated for the CMS experiment. The MSSM parameter space for $\mathrm{m}_{\mathrm{A}} \gtrsim 150-200 \mathrm{GeV} / c^{2}$ is expected to be covered with at least one decay channel with an integrated luminosity of $60 \mathrm{fb}^{-1}$. A light stop quark with large stop mixing can affect seriously the discovery potential in the $\mathrm{h} \rightarrow \gamma \gamma$ and $\mathrm{h} \rightarrow \mathrm{ZZ}^{*}$ decay channels.
\end{abstract}

\section{Introduction}

The Minimal Supersymmetric Standard Model (MSSM) contains five Higgs bosons: a light CP-even Higgs boson h, a heavy CP-even Higgs boson H, a CP-odd Higgs boson A and two charged Higgs bosons $\mathrm{H}^{ \pm}$. At tree-level the $\mathrm{h}(\mathrm{H})$ mass is bound to be below (above) the $\mathrm{Z}$ boson mass. The higher order corrections increase this upper (lower) bound, the largest possible value being about $135 \mathrm{GeV} / c^{2}[1]$. The fact that in the MSSM one Higgs boson is bound to be light gives a strong prediction for the mass region where the lightest Higgs boson might be seen.

The LEP and Tevatron results have already constrained the MSSM parameter space significantly. The measurements yield lower bounds of 91.0 and $91.9 \mathrm{GeV} / c^{2}$ for the lightest $\mathrm{CP}$-even Higgs boson $\mathrm{h}$ and for the $\mathrm{CP}$-odd $\mathrm{A}$, respectively [2]. The excluded $\tan \beta$ regions are $0.5<\tan \beta<2.4$ for the maximal $\mathrm{m}_{\mathrm{h}}$ scenario (maximal mixing scenario) and $0.7<\tan \beta<10.5$ for the no-stop-mixing scenario [2], assuming top quark mass $174.3 \mathrm{GeV} / \mathrm{c}^{2}$. The recent results from the Tevatron, however, give the world average for the top mass of $178.0 \pm 4.3 \mathrm{GeV} / c^{2}$ [3]. The larger top mass softens the bounds, for example assuming the top mass of $179.3 \mathrm{GeV} / \mathrm{c}^{2}$ the excluded region by LEP in the maximal mixing scenario is $0.9<\tan \beta<1.5$ and for top mass of about $183 \mathrm{GeV} / \mathrm{c}^{2}$ the exclusion vanishes [4]. Some constraints have been derived from

$\S$ On leave from ITEP, Moscow, Russia

\| Present address: Instituut-Lorentz, Leiden University, The Netherlands 
the existing data for the other SUSY parameters. The value of the trilinear coupling $\mathrm{A}_{\mathrm{t}}$ is limited to $350 \mathrm{GeV} / c^{2} \lesssim \mathrm{A}_{\mathrm{t}} \lesssim 1.5(2.3) \mathrm{TeV} / c^{2}$ for a light stop quark with $\mathrm{m}_{\tilde{\mathrm{t}}_{1}}=200(400) \mathrm{GeV} / c^{2}$ and for the experimental constraint $\mathrm{m}_{\mathrm{h}} \gtrsim 90 \mathrm{GeV} / c^{2}[5]$. The higgsino and gaugino mass parameters $\mu$ and $\mathrm{M}_{2}$ are related to neutralino and chargino masses, and experimental mass bounds can be used to exclude $|\mu|$ and $\mathrm{M}_{2}$ values below $100 \mathrm{GeV} / c^{2}$ [6]. The present experimental lower bound from LEP for the stop quark mass is $\sim 100 \mathrm{GeV} / c^{2}[7]$. The mass limit from the Tevatron RunII with the ultimate luminosities $\left(\sim 20 \mathrm{fb}^{-1}\right)$ is expected to reach $\mathrm{m}_{\tilde{t}_{1}} \sim 240 \mathrm{GeV} / c^{2}[8]$.

In this work three SUSY scenarios are considered: a no-mixing scenario where the mixing of the left and right handed stop eigenstates do not play any significant role and where all SUSY particles are assumed to be heavy [9], a maximal-mixing scenario which maximizes the $\mathrm{h}$ mass [9], and a light-stop scenario in which the stop quark mass is of the same order as the top quark mass [5]. These scenarios do not assume any particular model for the soft SUSY-breaking mechanism. The stop mixing parameter is defined as $\mathrm{X}_{\mathrm{t}}=\mathrm{A}_{\mathrm{t}}-\mu \cot \beta$, with $\mathrm{A}_{\mathrm{t}}$ being the trilinear Higgs-stop coupling. The stop-mixing is maximized when $X_{t}=\sqrt{6} \times M_{\text {SUSY }}$, where $M_{\text {SUSY }}$ is the heavy SUSY scale [9]. In this work, the maximal stop-mixing scenario is defined taking $A_{t}=\sqrt{6} \times M_{S U S Y}$, with $M_{\text {SUSY }}$ $=1 \mathrm{TeV} / c^{2}$. With respect to the standard definition, this choice leads to a deviation of less than $1 \%$ in the total production rate of $\mathrm{pp} \rightarrow \mathrm{h}+\mathrm{X}, \mathrm{h} \rightarrow \gamma \gamma$ at the LHC. No sbottom mixing is assumed taking $\mathrm{A}_{\mathrm{b}}=0$. The higgsino mass parameter $\mu$ is set to $300 \mathrm{GeV} / c^{2}$ and the gaugino mass parameter $\mathrm{M}_{2}$ is set to $200 \mathrm{GeV} / c^{2}$, values chosen large enough not to be already experimentally excluded. All the soft SUSY breaking mass parameters are set to $1 \mathrm{TeV} / c^{2}$, and the gluino mass $\mathrm{M}_{\tilde{\mathrm{g}}}$ is set to $800 \mathrm{GeV} / \mathrm{c}^{2}$. The mass of the top quark is set to $175 \mathrm{GeV} / c^{2}$. The values of these parameters are taken to be the same in the no-mixing scenario, except that of the trilinear coupling $\mathrm{A}_{\mathrm{t}}$ which is set to zero. For the light-stop scenario $\mathrm{A}_{\mathrm{t}}$ is taken to be $1400 \mathrm{GeV} / c^{2}$ close to the highest possible experimentally allowed value $\left(\mathrm{A}_{\mathrm{t}}=1500 \mathrm{GeV} / c^{2}\right)$ with light stop quarks [5]. In this scenario $\mu$ is set to $-250 \mathrm{GeV} / c^{2}$ and $\mathrm{M}_{2}$ to $250 \mathrm{GeV} / c^{2}$. The soft SUSY breaking mass parameters are set to $1 \mathrm{TeV} / c^{2}$ except the mass parameters of the stop sector, which are required to be of the order of $500 \mathrm{GeV} / c^{2}$ to allow the stop quark to be light. The actual value of the stop sector soft SUSY breaking mass parameters vary depending on the chosen stop quark mass.

With the above values of the SUSY parameters the upper bound of $m_{h}$ is about $127 \mathrm{GeV} / c^{2}$ in the maximal-mixing scenario and about $114 \mathrm{GeV} / c^{2}$ in the no-mixing scenario. The sign of $\mu$ has only a small effect on the mass of the lightest Higgs boson. In the light-stop scenario with $\mathrm{m}_{\tilde{\mathrm{t}}_{1}}=200 \mathrm{GeV} / c^{2}$, the upper bound of $\mathrm{m}_{\mathrm{h}}$ is $113 \mathrm{GeV} / c^{2}$, as in the no-mixing scenario. For $\mathrm{m}_{\tilde{\mathrm{t}}_{1}}=300 \mathrm{GeV} / c^{2}$ this upper bound increases by $10 \mathrm{GeV} / c^{2}$ approaching to that of the maximal-mixing scenario.

The $\mathrm{H} \rightarrow \gamma \gamma$ channel is considered one of the major discovery channels for a light Standard Model (SM) Higgs boson and for the lightest scalar MSSM Higgs boson at the LHC. There can be, however, regions of the MSSM parameter space where this discovery potential is reduced. The effect of a light stop quark in the presence of large mixing 
has been calculated and the consequences for the gg $\rightarrow \mathrm{h} \rightarrow \gamma \gamma$ channel have been discussed in Ref. [5]. An experimental study was performed in Ref. [10] and the discovery potential was calculated for the $\mathrm{h} \rightarrow \gamma \gamma$ channel in the CMS detector. In this earlier work, however, only the gluon-gluon fusion production process was simulated and other Higgs boson decay channels were not considered. In the present work, all significant production processes are included in the calculation of the inclusive $\mathrm{h} \rightarrow \gamma \gamma$ rate in the MSSM and the discovery potential is evaluated also in the associated production and weak gauge boson fusion production processes qq $\rightarrow$ qqh. Furthermore, updated programs are used to calculate the cross sections and branching ratios.

The aim of this paper is to extend the study of the loop effects in the $\mathrm{h} \rightarrow \gamma \gamma$ channel to the full discovery potential of the lightest scalar Higgs boson at the LHC. Therefore, the $\mathrm{h} \rightarrow \mathrm{ZZ}^{*} \rightarrow \ell^{+} \ell^{-} \ell^{\prime+} \ell^{\prime-}$ and $\mathrm{h} / \mathrm{H} \rightarrow \tau^{+} \tau^{-}$decay channels were also studied. The $\mathrm{h} \rightarrow \mathrm{ZZ}^{*} \rightarrow \ell^{+} \ell^{-} \ell^{\prime+} \ell^{\prime-}$ has not been so far considered as a discovery channel in the MSSM at large $\tan \beta$. It is shown, however, in Section 3.2 that this channel can yield a large discovery potential if the SUSY scenario is such that $\mathrm{m}_{\mathrm{h}}^{\max } \gtrsim 125 \mathrm{GeV} / c^{2}$. The $\mathrm{h} / \mathrm{H} \rightarrow \tau^{+} \tau^{-}$decay channels with lepton+jet and two-lepton final states in the weak gauge boson fusion production have been shown to be particularly interesting and to cover the full of the MSSM parameter space [11]. In this paper the discovery potential for this channel is calculated with realistic detector sensitivities. The CMS detector sensitivities are used and were obtained from the recent simulations for the discovery potential of a light SM Higgs boson [12].

\section{Phenomenology}

\subsection{Production cross sections}

The lightest MSSM Higgs boson $\mathrm{h}$ is produced through the gluon fusion gg $\rightarrow \mathrm{h}$, the associated processes $\mathrm{q} \overline{\mathrm{q}} / \mathrm{gg} \rightarrow \mathrm{t} \overline{\mathrm{t}}, \mathrm{q} \overline{\mathrm{q}} / \mathrm{gg} \rightarrow \mathrm{b} \overline{\mathrm{b}} \mathrm{h}, \mathrm{qq} \rightarrow \mathrm{Wh} / \mathrm{Zh}$ and through the weak gauge boson fusion process qq $\rightarrow$ qqh. The gluon fusion process dominates the production over the entire parameter space. This process is mediated by heavy quark and squark triangle loops. The cross sections to the leading order (LO) and to the next to leading order (NLO) are calculated in this work with the program HIGLU [13]. The top and bottom loops are included in the calculation of the Higgs boson coupling to gluons in this program. Since the squark loops are not included, the decay width $\Gamma(\mathrm{h} \rightarrow \mathrm{gg})$, calculated with the HDECAY program [14], is used to include the squark loop effects: the cross section given by HIGLU is divided by the decay width $\Gamma(\mathrm{h} \rightarrow \mathrm{gg})$ with sparticle loops switched off, and multiplied by the decay width with all sparticle effects. The Higgs boson mass is kept constant in this procedure. The corrected gluon fusion cross section with SUSY loop effects can be presented with the respective branching ratios and total widths as

$$
\sigma \cdot \mathrm{BR}=\sigma(\mathrm{gg} \rightarrow \mathrm{h}) \cdot \frac{\mathrm{BR}(\mathrm{h} \rightarrow \mathrm{gg})^{\text {susy }}}{\mathrm{BR}(\mathrm{h} \rightarrow \mathrm{gg})^{\text {nosusy }}} \frac{\Gamma_{\mathrm{TOT}}^{\text {susy }}}{\Gamma_{\mathrm{TOT}}^{\text {nousy }}} \cdot \mathrm{BR}(\mathrm{h} \rightarrow \gamma \gamma)_{\text {susy }}
$$


Table 1. Production cross sections for the lightest MSSM Higgs boson for $\mathrm{m}_{\mathrm{h}}=125.8 \mathrm{GeV} / c^{2}\left(\mathrm{~m}_{\mathrm{A}}=250 \mathrm{GeV} / c^{2}\right)$ and $\tan \beta=10$ with maximal stop mixing.

\begin{tabular}{|c|c|c|c|c|c|c|c|}
\hline process & $\mathrm{gg} \rightarrow \mathrm{h}$ & $\mathrm{qq} \rightarrow \mathrm{qqh}$ & $\mathrm{qq} \rightarrow \mathrm{Wh}$ & $\mathrm{qq} \rightarrow \mathrm{Zh}$ & $\mathrm{pp} \rightarrow \mathrm{b} \overline{\mathrm{bh}}$ & $\mathrm{pp} \rightarrow \mathrm{t} \overline{\mathrm{th}}$ & $\sigma_{\mathrm{TOT}}$ \\
\hline$\sigma(\mathrm{pb})$ & 27.3 & 4.17 & 1.59 & 0.64 & 0.72 & 0.32 & 34.1 \\
\hline$\sigma / \sigma_{\mathrm{TOT}}$ & $79 \%$ & $12 \%$ & $4.6 \%$ & $1.8 \%$ & $2.1 \%$ & $0.9 \%$ & $100 \%$ \\
\hline
\end{tabular}

where nosusy refers to the branching ratio and total width calculated assuming heavy SUSY particles and susy to the same variables with SUSY spectrum determined by the given scenario.

The cross sections for the associated production with weak gauge bosons qq $\rightarrow$ Wh and qq $\rightarrow$ Zh are calculated with the program V2HV [15] to both leading and next to leading order. The cross sections for the production with associated quark pairs $\mathrm{q} \overline{\mathrm{q}} / \mathrm{gg} \rightarrow \mathrm{t} \overline{\mathrm{th}}$ and $\mathrm{q} \overline{\mathrm{q}} / \mathrm{gg} \rightarrow \mathrm{b} \overline{\mathrm{b}} \mathrm{g}$ are calculated with the HQQ program [15], which presently includes only the LO processes. The cross sections for the weak gauge boson fusion qq $\rightarrow$ qqh are evaluated with the VV2H program [15]. The production cross sections and the contributions from the individual production processes to the total cross section at $\mathrm{m}_{\mathrm{h}}=125.8 \mathrm{GeV} / \mathrm{c}^{2}\left(\mathrm{~m}_{\mathrm{A}}=250 \mathrm{GeV} / \mathrm{c}^{2}\right), \tan \beta=10$ are shown in Table 1. The gluon fusion process contributes about $80 \%$ to the total cross section. This fraction is not sensitive to the Higgs boson mass and $\tan \beta$.

In the $\mathrm{SM}$, the $\mathrm{K}$-factor (defined as $\mathrm{K}=\sigma_{\mathrm{NLO}} / \sigma_{\mathrm{LO}}$ ) for the gluon fusion process is large varying between 1.5 and 1.7 [16]. In the MSSM, this K-factor depends on $\tan \beta$ being about the same as in the SM at small $\tan \beta$ and closer to unity at large $\tan \beta$ [16]. The K-factors do not depend significantly on the squark mass and are stable against the loop effects in the gluon fusion mechanism even in the extreme situation when one of the stop mass eigenstates is light while the other squarks are heavy and decouple [17]. The K-factor in the associated process qq $\rightarrow$ Wh is almost independent of $\mathrm{m}_{\mathrm{A}}$ and $\tan \beta$ and is about 1.3 for both the no-mixing and maximal-mixing scenario.

\subsection{Decay channels}

Figures 1 and 2 show the branching ratios for the lightest MSSM Higgs boson as a function of $\mathrm{m}_{\mathrm{A}}$ and $\mathrm{m}_{\mathrm{h}}$ with maximal stop quark mixing for $\tan \beta=10$ and 30 , respectively. The branching ratios and decay widths are calculated with the program HDECAY 3.0 [14]. The next to leading order (NLO) values are used for the decay modes throughout this study. The $\mathrm{h} \rightarrow \mathrm{b} \overline{\mathrm{b}}$ decay channel dominates. The branching ratios to weak gauge bosons $\mathrm{h} \rightarrow \mathrm{ZZ}^{*}$ and $\mathrm{h} \rightarrow \mathrm{WW}^{*}$ increase rapidly when $\mathrm{m}_{\mathrm{h}}$ approaches its maximum value reaching $\sim 2 \%$ and $\sim 20 \%$, respectively, at large $\mathrm{m}_{\mathrm{A}}$. For $\mathrm{m}_{\mathrm{A}} \gtrsim 200 \mathrm{GeV} / c^{2}$ the branching ratio for the $\mathrm{h} \rightarrow \gamma \gamma$ decay channel is between one and two per mil. The branching ratios for the $\mathrm{h} \rightarrow \tau^{+} \tau^{-}, \mathrm{h} \rightarrow \mathrm{b} \overline{\mathrm{b}}$ and $\mathrm{h} \rightarrow \mu^{+} \mu^{-}$ decay channels remain large also for $\mathrm{m}_{\mathrm{A}} \lesssim 200 \mathrm{GeV} / c^{2}$, where the lightest Higgs boson is not SM-like, due to the enhanced couplings to the down type fermions. 


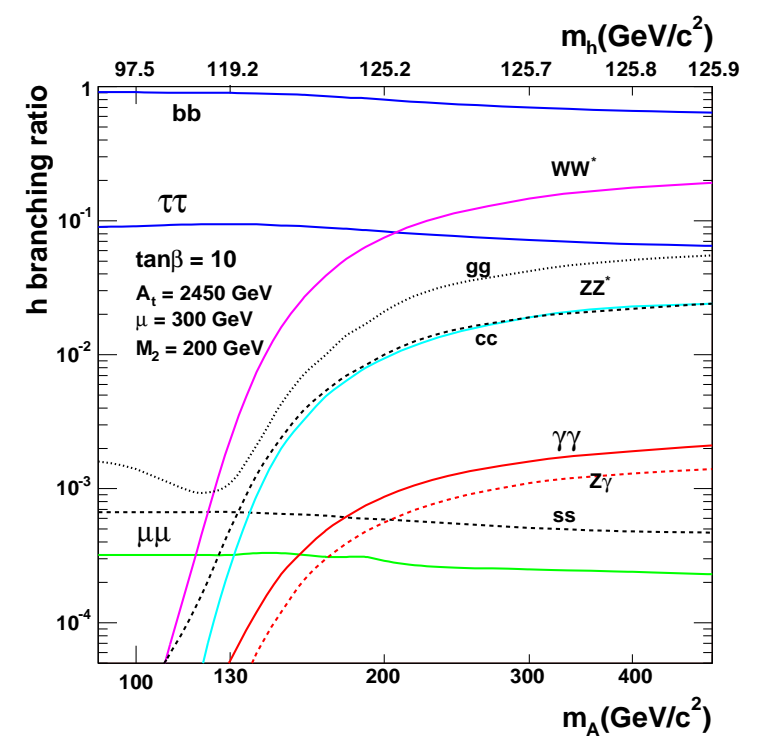

Figure 1. Branching ratios for the lightest MSSM Higgs boson as a function of $\mathrm{m}_{\mathrm{A}}$ and $\mathrm{m}_{\mathrm{h}}$ for $\tan \beta=10$ with maximal stop quark mixing.

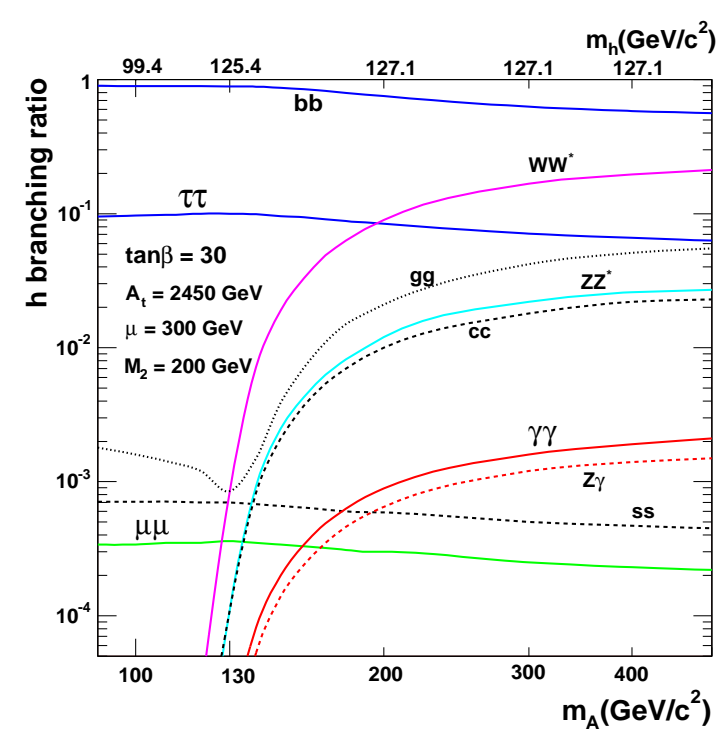

Figure 2. The same as in Fig. 1 but for $\tan \beta=30$.

\subsection{Effect of SUSY parameters}

In the MSSM, one of the stop quarks may become much lighter than the other squarks if mixing between different squark isospin eigenstates is large. The mixing can be described with the following mass matrix [18]

$$
\left(\begin{array}{cc}
\mathrm{m}_{\tilde{t}_{\mathrm{L}}}^{2} & \mathrm{~m}_{\text {top }}\left(\mathrm{A}_{\mathrm{t}}-\mu \cot \beta\right) \\
\mathrm{m}_{\mathrm{top}}\left(\mathrm{A}_{\mathrm{t}}-\mu \cot \beta\right) & \mathrm{m}_{\tilde{\mathrm{t}}_{\mathrm{R}}}^{2}
\end{array}\right)
$$

where $\tilde{t}_{L}$ and $\tilde{t}_{R}$ are the left and right handed eigenstates of the stop quark and $\mathrm{A}_{\mathrm{t}}-\mu \cot \beta \equiv \mathrm{X}_{\mathrm{t}}$ is the squark mixing parameter with $\mathrm{A}_{\mathrm{t}}$ being the trilinear coupling and $\mu$ the higgsino mass parameter. Mixing in the third generation squark sector may be important, since, as seen from the off-diagonal terms of the mass matrix, the squark mixing is proportional to the corresponding quark mass. For a heavy top quark, mixing in the stop sector may thus produce considerable splitting between the mass eigenstates $\tilde{\mathrm{t}}_{1}, \tilde{\mathrm{t}}_{2}$

$$
\mathrm{m}_{\mathfrak{\mathrm { t }}_{1,2}}^{2}=\frac{1}{2}\left(\mathrm{~m}_{\tilde{\mathrm{t}}_{\mathrm{L}}}^{2}+\mathrm{m}_{\tilde{\mathrm{t}}_{\mathrm{R}}}^{2}\right) \mp \frac{1}{2} \sqrt{\left(\mathrm{m}_{\tilde{\mathrm{t}}_{\mathrm{L}}}^{2}-\mathrm{m}_{\tilde{\mathrm{t}}_{\mathrm{R}}}^{2}\right)^{2}+4 \mathrm{~m}_{\mathrm{top}}^{2}\left(\mathrm{~A}_{\mathrm{t}}-\mu \cot \beta\right)^{2}}
$$

resulting in one very light and one very heavy stop quark. In above relations $\mathrm{m}_{\tilde{\mathrm{t}}_{\mathrm{L}}}^{2}=$ $\mathrm{M}_{\tilde{\mathrm{Q}}}^{2}+\mathrm{m}_{\mathrm{Z}}^{2} \cos 2 \beta\left(\mathrm{I}_{3}^{\tilde{\mathrm{t}}}-\mathrm{e}_{\mathrm{t}} \sin ^{2} \theta_{\mathrm{W}}\right)+\mathrm{m}_{\text {top }}^{2}$ and $\mathrm{m}_{\tilde{\mathrm{t}}_{\mathrm{R}}}^{2}=\mathrm{M}_{\tilde{\mathrm{U}}}^{2}+\mathrm{m}_{\mathrm{Z}}^{2} \cos 2 \beta \mathrm{e}_{\tilde{\mathrm{t}}} \sin ^{2} \theta_{\mathrm{W}}+\mathrm{m}_{\text {top }}^{2}$ where $\mathrm{M}_{\tilde{Q}}$ and $\mathrm{M}_{\tilde{U}}$ are the soft-SUSY breaking scalar masses, $\mathrm{I}_{3}^{\tilde{t}}$ is the squark weak isospin and $e_{\tilde{t}}$ the squark charge. It can be seen that in order to have large splitting between the two stop eigenstates and therefore a light stop quark, $\mathrm{X}_{\mathrm{t}}$ must be large. For a common scalar mass parameter of $1 \mathrm{TeV}$, the mass of the lighter stop quark is of the order of 
$800 \mathrm{GeV} / c^{2}$. A light stop quark $\left(\mathrm{m}_{\text {stop }} \lesssim 300 \mathrm{GeV} / c^{2}\right)$ can be obtained choosing the third generation scalar masses $\mathrm{M}_{\tilde{U}}$ and $\mathrm{M}_{\tilde{\mathrm{Q}}}$ to be of the order of $500 \mathrm{GeV} / c^{2}$. For these parameter values, the mass of the lightest supersymmetric particle LSP is of the order of $100 \mathrm{GeV} / \mathrm{c}^{2}$, well above the present experimental limit [2]. In the next sections, the lighter of the two stop mass eigenstates is denoted simply as a stop quark, stop $\equiv \tilde{\mathrm{t}}_{1}$ and its mass $\mathrm{m}_{\text {stop }}$.

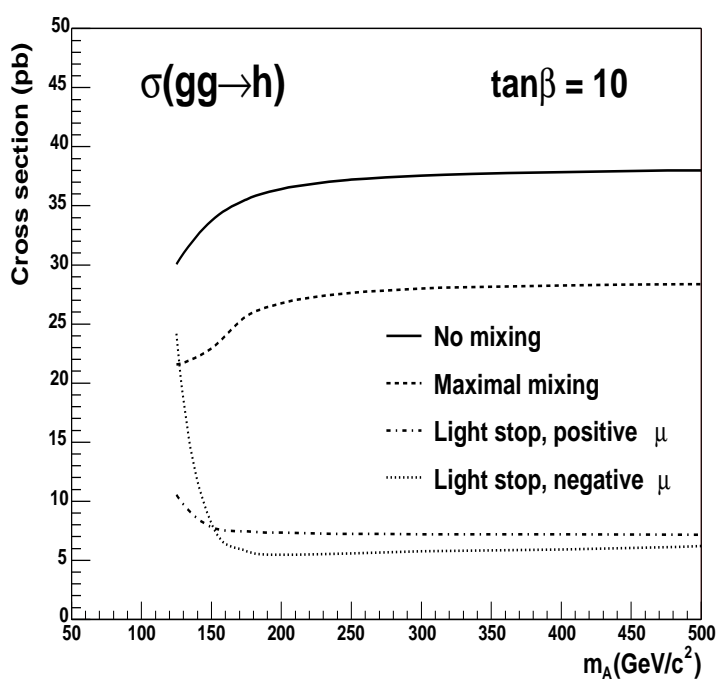

Figure 3. Cross section for the gg $\rightarrow$ h process with $\tan \beta=10$ without stop mixing, with maximal stop mixing and with light stop quark $\mathrm{m}_{\text {stop }}=200 \mathrm{GeV} / c^{2}$ for $\mu<0$ and for $\mu>0$.

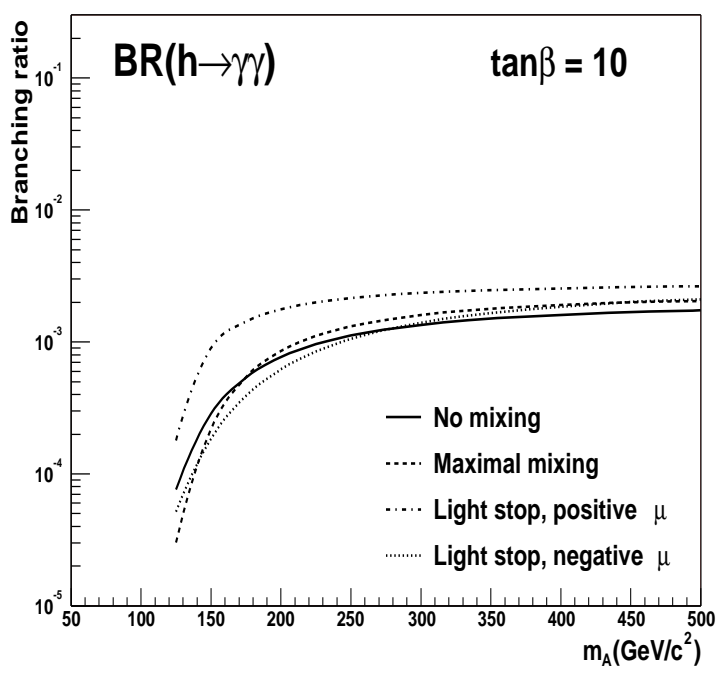

Figure 4. The branching ratio for the $\mathrm{h} \rightarrow \gamma \gamma$ decay with $\tan \beta=10$ without stop mixing, with maximal stop mixing and with light stop quark $\mathrm{m}_{\text {stop }}=200 \mathrm{GeV} / c^{2}$ for $\mu<0$ and for $\mu>0$.

Since no supersymmetric particles have been found so far, the supersymmetry must be broken, the squarks do not have the same masses as quarks and the cancellation at loop level is less significant. The results from existing experiments indicate that a scenario with large mixing in the squark (stop) sector is possible and more likely than a no-mixing scenario [2]. If the mass of the stop quark is small, of the order of the top quark mass, the cancellation effects become important.

It has been first shown in Ref. [5] that the rate for the gg $\rightarrow \mathrm{h} \rightarrow \gamma \gamma$ process could be strongly reduced with large mixing and with a light stop quark $\left(\mathrm{m}_{\text {stop }} \sim \mathrm{m}_{\text {top }}\right)$. The top - stop interference leads to a suppression of the top quark contribution in the loops mediating the Higgs boson production, since the stop loop interferes destructively with the top quark and the top and stop loops partly cancel. The loop mediated Higgs boson decay into photons is also affected, but since the dominant contribution comes now from a $\mathrm{W}$ loop, which interferes destructively with the top loop, a reduction of the top contribution by interfering stop loops increases the $h \rightarrow \gamma \gamma$ partial width. As the 
W loop dominates in this partial width, the interference effect is smaller than in the $\mathrm{gg} \rightarrow \mathrm{h}$ process dominated by a top quark loop. In addition to the light stop, there are contributions from the charged Higgs bosons, sfermions and especially from charginos, but their net effect to the $\mathrm{h} \rightarrow \gamma \gamma$ partial width is small, less than $\sim 10 \%$ [19]. At large $\tan \beta$ also the bottom loop contributes and even becomes larger than the top loop contribution [20]. As the reduction of the gg $\rightarrow \mathrm{h}$ partial width is significantly stronger than the enhancement of the $\mathrm{h} \rightarrow \gamma \gamma$ partial width, the rate for $\mathrm{gg} \rightarrow \mathrm{h} \rightarrow \gamma \gamma$ is reduced. For $\mathrm{m}_{\mathrm{A}} \gtrsim 100 \mathrm{GeV} / c^{2}, \mathrm{~A}_{\mathrm{t}}=1.5 \mathrm{TeV} / c^{2}, \mathrm{~m}_{\text {stop }}=200 \mathrm{GeV} / c^{2}$ and $\mu=300 \mathrm{GeV} / c^{2}$ the rate is reduced by a factor of $\sim 10$ relative to the no-mixing scenario with heavy SUSY particles. The squark sector can affect the branching ratios of the lightest Higgs boson only via this interference phenomenon because the decays into gauginos are not kinematically allowed.

Figure 3 shows the cross section for the gg $\rightarrow$ h process and Fig. 4 the branching ratios for the $\mathrm{h} \rightarrow \gamma \gamma$ decay channel corrected for the loop effects for the following scenarios: no stop mixing, maximal stop mixing, light stop quark $\mathrm{m}_{\text {stop }}=200 \mathrm{GeV} / \mathrm{c}^{2}$ with $\mu<0$ and light stop quark $\mathrm{m}_{\text {stop }}=200 \mathrm{GeV} / c^{2}$ with $\mu>0$. The interference between the stop and top quarks is clearly visible: the lighter the stop quark the stronger the interference and smaller the cross section.

At large $\tan \beta$ the $b$ couplings are enhanced, but the contributions from the sbottom loops are suppressed compared to the bottom loops by $\left(\mathrm{m}_{\mathrm{b}} / \mathrm{m}_{\tilde{\mathrm{b}}}\right)^{2}[17]$. With the LEP lower bound for the sbottom mass [21], the contribution is on a per mille level. In this work the mixing in the sbottom sector is not considered.

\subsection{Search strategies}

In the expected mass range, $\mathrm{m}_{\mathrm{h}} \lesssim 135 \mathrm{GeV} / \mathrm{c}^{2}$, the lightest MSSM Higgs boson $\mathrm{h}$ can be searched for through the following decay channels: $\mathrm{h} \rightarrow \gamma \gamma \mathrm{h} \rightarrow \gamma \mathrm{Z}, \mathrm{h} \rightarrow \mu^{+} \mu^{-}$, $\mathrm{h} \rightarrow \mathrm{b} \overline{\mathrm{b}}, \mathrm{h} \rightarrow \mathrm{ZZ}^{*} \rightarrow \ell^{+} \ell^{-} \ell^{\prime+} \ell^{\prime-}, \mathrm{h} \rightarrow \mathrm{WW}^{*} \rightarrow \ell^{+} \ell^{-} \nu_{\ell} \nu_{\ell}$ and $\mathrm{h} \rightarrow \tau^{+} \tau^{-}$. The searches in the $\mathrm{h} \rightarrow \gamma \gamma, \mathrm{h} \rightarrow \mu^{+} \mu^{-}$and $\mathrm{h} \rightarrow \mathrm{ZZ}^{*} \rightarrow \ell^{+} \ell^{-} \ell^{\prime+} \ell^{\prime-}$ channels are based on the small total width of the Higgs boson in this mass range (in the SM and MSSM) exploiting the precise photon energy and lepton momentum measurements for the Higgs boson mass reconstruction $[22,23,24]$. The $\mathrm{h} \rightarrow \gamma \gamma$ and $\mathrm{h} \rightarrow \mathrm{ZZ}^{*} \rightarrow \ell^{+} \ell^{-} \ell^{\prime+} \ell^{\prime-}$ channels are expected to yield their largest reaches in the inclusive production, dominated by the gluon fusion process. The $\mathrm{h} \rightarrow \gamma \gamma$ channel can be searched for also in the associated production processes tth and Wh with a requirement of an isolated lepton from the $\mathrm{W} \rightarrow \ell \nu_{\ell}$ decay [25]. The signal-to-background ratios are larger but the event rates are smaller than for the inclusive production. For the $h \rightarrow b \bar{b}$ decay channel, suppression of the QCD multi-jet background is possible only in the associated production processes t $\overline{t h}$ and $\mathrm{Wh}$ with a requirement of an isolated lepton from the $\mathrm{W} \rightarrow \ell \nu_{\ell}$ decay [26]. The $\mathrm{h} \rightarrow \gamma \gamma, \mathrm{h} \rightarrow \mu^{+} \mu^{-}, \mathrm{h} \rightarrow \mathrm{WW}^{*} \rightarrow \ell^{+} \ell^{-} \nu_{\ell} \nu_{\ell}, \mathrm{h} \rightarrow \tau^{+} \tau^{-}$and possibly $\mathrm{h} \rightarrow \mathrm{b} \overline{\mathrm{b}}$ decay channels can be searched for also in the weak gauge boson fusion production process $\mathrm{qq} \rightarrow \mathrm{qqh}$. In this production mechanism tagging of the forward jets and vetoing on 
central hadronic jets can be used to efficiently suppress the QCD multi-jet, $\mathrm{W}+$ jets and tẼ backgrounds [27]. The $\mathrm{h} \rightarrow \tau^{+} \tau^{-}$channel is particularly interesting in the MSSM as the couplings to down type fermions are $\tan \beta$ enhanced relative to SM couplings. Due to the tiny branching ratios, the $\mathrm{h} \rightarrow \gamma \mathrm{Z}$ and $\mathrm{h} \rightarrow \mu^{+} \mu^{-}$decay channels may be exploited only with the integrated luminosities exceeding $100 \mathrm{fb}^{-1}$.

\section{Inclusive production channels}

\section{1. $\mathrm{h} \rightarrow \gamma \gamma$}

The isorate (cross section times branching ratio) curves for the $\mathrm{h} \rightarrow \gamma \gamma$ channel in the inclusive production in the no-mixing scenario are shown in Fig. 5 with LO cross sections and in Fig. 6 with NLO cross sections. The isorate curves for the inclusive production in the maximal-mixing scenario are shown in Fig. 7 with LO and in Fig. 8 with NLO cross sections. Due to the larger Higgs boson mass $\mathrm{m}_{\mathrm{h}}$ in the maximal-mixing scenario for fixed $\mathrm{m}_{\mathrm{A}}$ and $\tan \beta$ the cross section is smaller than that in the no-mixing scenario. This decrease is compensated by a larger $\mathrm{h} \rightarrow \gamma \gamma$ branching ratio resulting in $\mathrm{a} \sim 3 \%$ lower production (cross section times branching ratio) rate relative to the no-mixing scenario. Although in this scenario the stop quark is rather heavy $\mathrm{m}_{\text {stop }} \simeq 800 \mathrm{GeV} / c^{2}$, the effect of the virtual stop loops suppresses the cross section by approximately $10 \%$ relative to the no-mixing scenario. A negative higgsino mass parameter would yield a further small suppression.

Figure 9 shows the cross section times branching ratio required for a $5 \sigma$ statistical significance in the inclusive $\mathrm{h} \rightarrow \gamma \gamma$ channel as a function of the invariant two-photon mass for 30 and $100 \mathrm{fb}^{-1}$ in the CMS detector [22]. The NLO cross sections are assumed for the signal and backgrounds. In the mass range of the lightest MSSM Higgs boson, $\mathrm{m}_{\mathrm{h}} \lesssim 127 \mathrm{GeV} / \mathrm{c}^{2}$, production rates at least 55 and $33 \mathrm{fb}$ are needed to obtain a $5 \sigma$ statistical significance with an integrated luminosities of 30 and $100 \mathrm{fb}^{-1}$, respectively. In the no-mixing scenario with $\mathrm{m}_{\mathrm{h}} \lesssim 114 \mathrm{GeV} / c^{2}$ the minimal production rates required for these luminosities are 71 and $42 \mathrm{fb}$, respectively. Larger rates are needed at lower mass values due to the increasing backgrounds. With these detector sensitivities a $5 \sigma$-discovery potential is expected for $\mathrm{m}_{\mathrm{A}} \gtrsim 200$ and $300 \mathrm{GeV} / c^{2}$ with integrated luminosities of 100 and $30 \mathrm{fb}^{-1}$, respectively. The reach is approximately the same in the no-mixing and maximal-mixing scenario.

Figures 10, 11 and 12 show the isorate curves for the $\mathrm{h} \rightarrow \gamma \gamma$ channel in the light-stop scenario. Figure 10 shows the isorate curves for the dominating gluon fusion process which is affected most by a light stop quark. Figure 11 shows the isorate curves in the inclusive production for a very light stop quark, $m_{\text {stop }}=200 \mathrm{GeV} / c^{2}$. Since the gluon fusion process is the dominating production mechanism, the effect of a light stop on the inclusive production is large, too. A discovery in the inclusive $\mathrm{h} \rightarrow \gamma \gamma$ channel with such a light stop quark could be possible only for $\mathrm{m}_{\mathrm{A}} \gg 500 \mathrm{GeV} / c^{2}$ for integrated luminosities exceeding $100 \mathrm{fb}^{-1}$. Figure 12 shows the isorate curves for the 


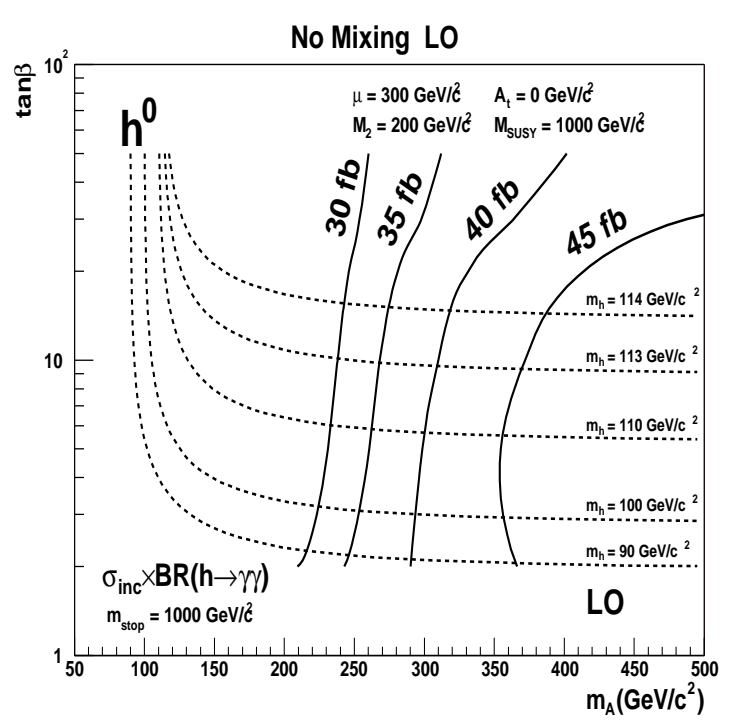

Figure 5. Isorate (cross section times branching ratio) curves for the inclusive $\mathrm{h} \rightarrow \gamma \gamma$ channel in the no-mixing scenario with LO cross sections. The isomass curves for the lightest MSSM Higgs boson are shown with dashed lines.

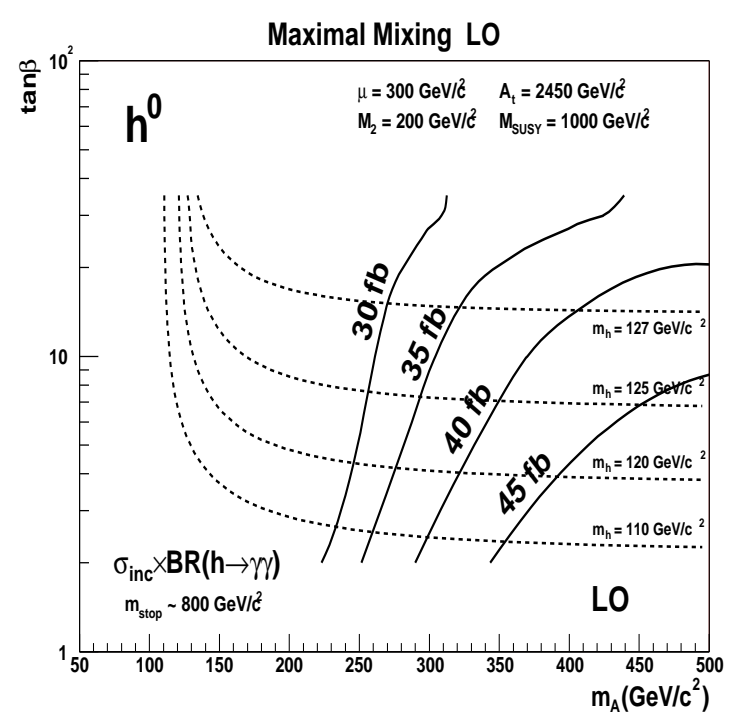

Figure 7. Isorate curves for the inclusive $\mathrm{h} \rightarrow \gamma \gamma$ channel in the maximal-mixing scenario with LO cross sections.

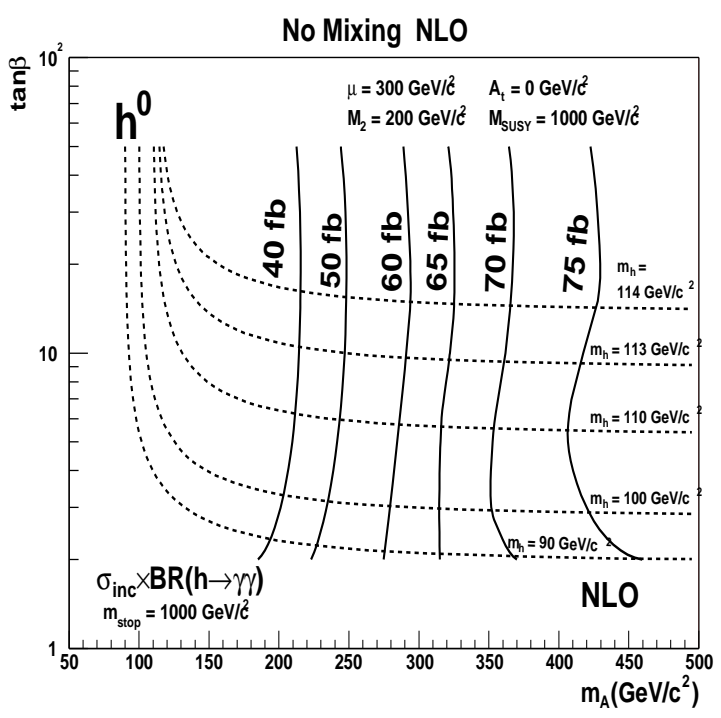

Figure 6. Isorate (cross section times branching ratio) curves for the inclusive $\mathrm{h} \rightarrow \gamma \gamma$ channel in the no-mixing scenario with NLO cross sections. The isomass curves for the lightest MSSM Higgs boson are shown with dashed lines.

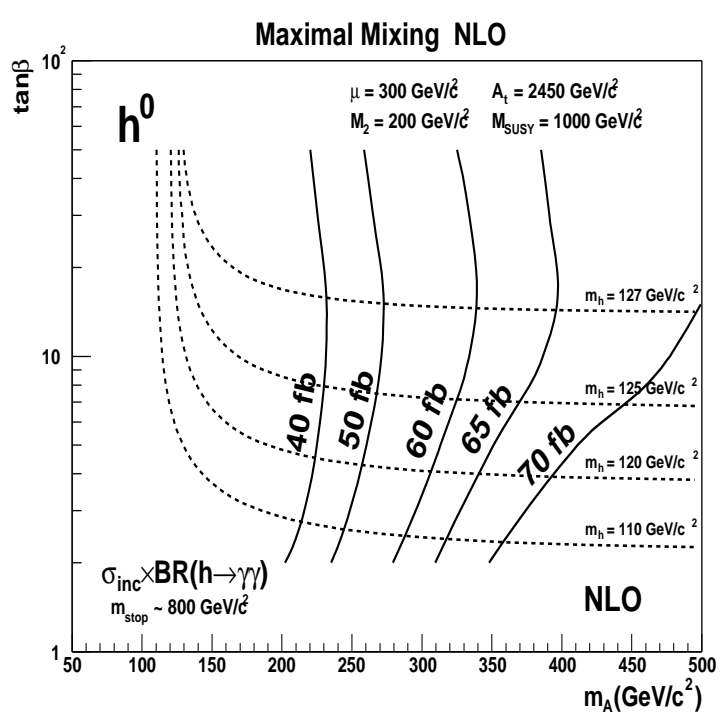

Figure 8. Isorate curves for the inclusive $\mathrm{h} \rightarrow \gamma \gamma$ channel in the maximal-mixing scenario with NLO cross sections.

inclusive production with $\mathrm{m}_{\text {stop }}=300 \mathrm{GeV} / \mathrm{c}^{2}$. For this value of $\mathrm{m}_{\text {stop }}$ a discovery is possible with $100 \mathrm{fb}^{-1}$ in part of the parameter space for about $\mathrm{m}_{\mathrm{A}} \gtrsim 400 \mathrm{GeV} / c^{2}$ and 


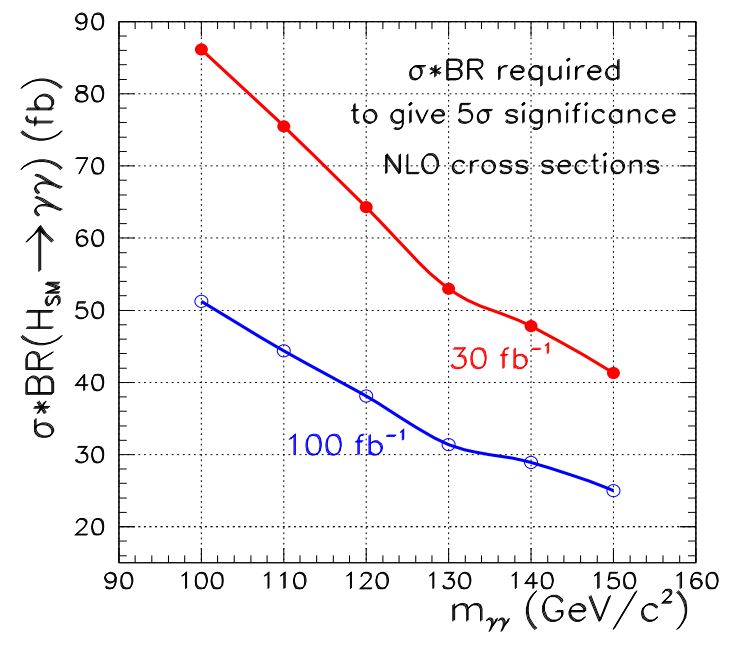

Figure 9. Cross section times branching ratio to give a $5 \sigma$ statistical significance for the inclusive $\mathrm{H} \rightarrow \gamma \gamma$ channel in the SM for 30 and $100 \mathrm{fb}^{-1}$ assuming NLO cross sections for the backgrounds [22].

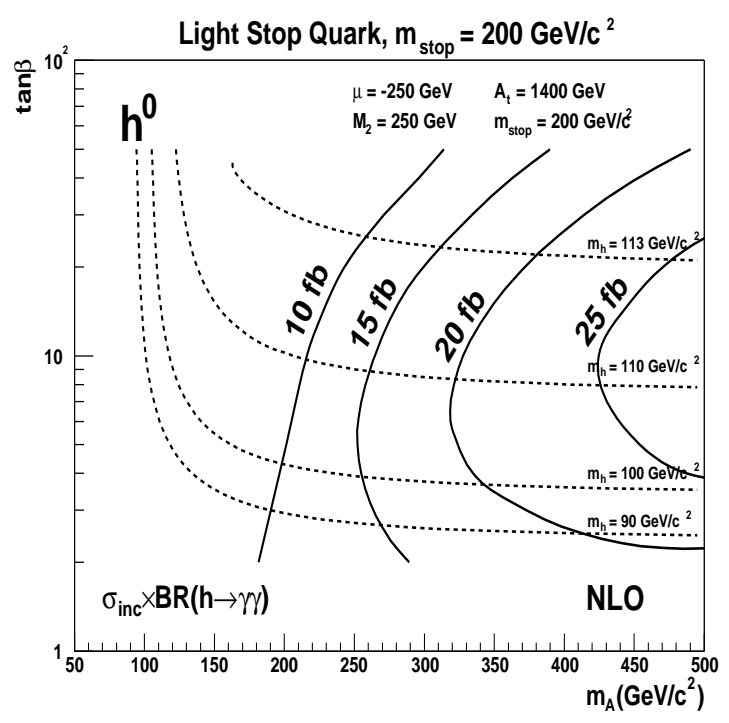

Figure 11. Isorate curves for the inclusive $\mathrm{h} \rightarrow \gamma \gamma$ channel with a light stop quark $\mathrm{m}_{\text {stop }}=200 \mathrm{GeV} / c^{2}$ with $\mathrm{NLO}$ cross sections.

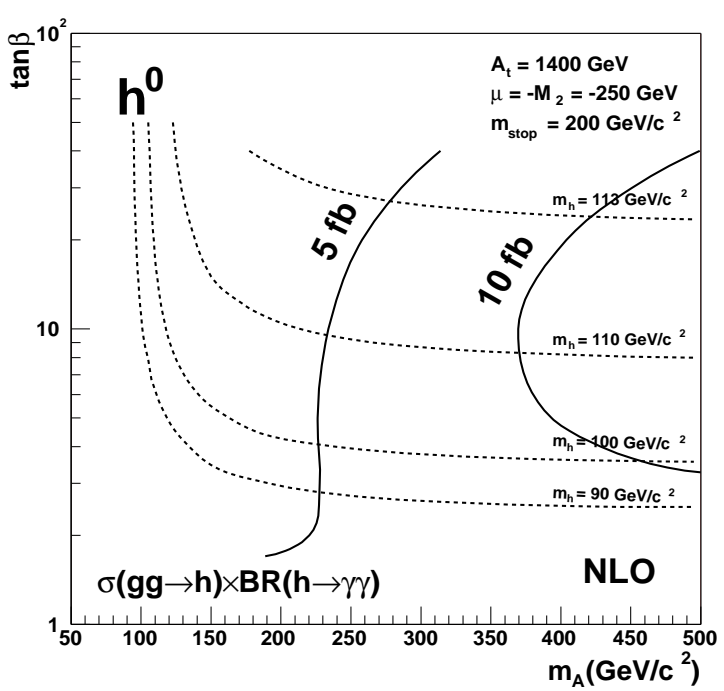

Figure 10. Isorate curves for the $\mathrm{gg} \rightarrow \mathrm{h} \rightarrow \gamma \gamma$ channel with a light stop quark $\mathrm{m}_{\text {stop }}=200 \mathrm{GeV} / c^{2}$ with $\mathrm{LO}$ cross sections.

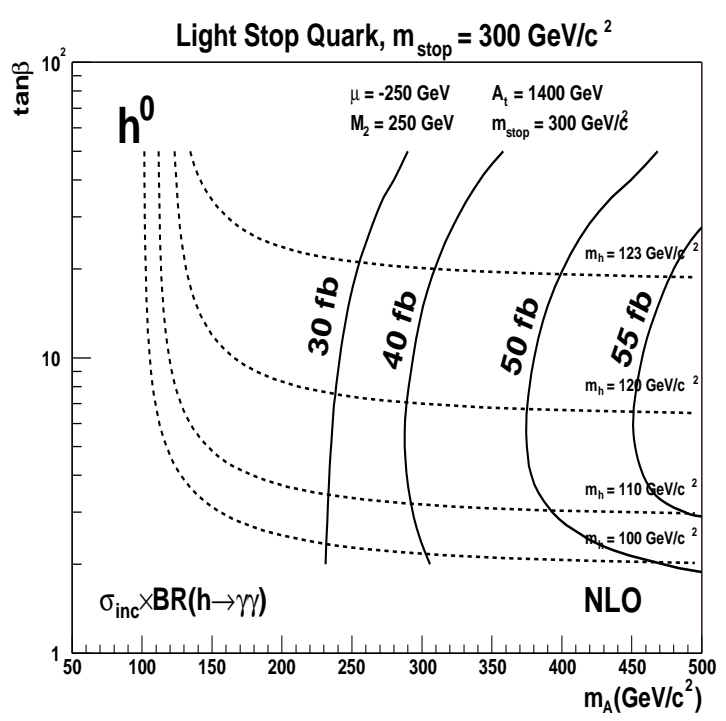

Figure 12. Isorate curves for the inclusive $\mathrm{h} \rightarrow \gamma \gamma$ channel with a light stop quark $\mathrm{m}_{\text {stop }}=300 \mathrm{GeV} / c^{2}$ with $\mathrm{NLO}$ cross sections.

$\tan \beta \gtrsim 10$. For $\mathrm{m}_{\text {stop }} \gtrsim 400 \mathrm{GeV} / c^{2}$ the interference effect is already small and the rate is close to that of the no-mixing and maximal-mixing scenarios. 
3.2. $\mathrm{h} \rightarrow \mathrm{ZZ}^{*} \rightarrow \ell^{+} \ell^{-} \ell^{\prime+} \ell^{\prime-}$

The four-lepton channel, the $\mathrm{H} \rightarrow \mathrm{ZZ}^{*} \rightarrow \ell^{+} \ell^{-} \ell^{\prime+} \ell^{\prime-}$, has been shown to be the major discovery channel over the large mass rangion in the SM [12]. In the MSSM, the heavier scalar $\mathrm{H}$ could be searched for in the four-lepton channel at small $\tan \beta$. For $\mathrm{m}_{\mathrm{H}} \lesssim 2 \mathrm{~m}_{\mathrm{Z}}$, where the detector resolution dominates, the discovery potential could be obtained from that for the SM Higgs boson while for $\mathrm{m}_{\mathrm{H}} \gtrsim 2 \mathrm{~m}_{\mathrm{Z}}$ dedicated studies are needed due to the difference of total Higgs boson widths between the SM and MSSM in this region. For

the lighter scalar h, a discovery could be possible close to the maximal possible value of $\mathrm{m}_{\mathrm{h}}$ at large $\tan \beta$ and $\mathrm{m}_{\mathrm{A}}$. The discovery potential is strongly dependent on the lowest possible mass value accessible in the (pure) SM scenario, due to the fast decreasing $\mathrm{h} \rightarrow \mathrm{ZZ}^{*}$ branching ratio. The CMS studies have shown that this value could be as low as $\mathrm{m}_{\mathrm{H}} \sim 120 \mathrm{GeV} / \mathrm{c}^{2}$ with an integrated luminosity of $100 \mathrm{fb}^{-1}$ combining the electron and muon channels [28, 29]. Therefore a significant region at large $\tan \beta \operatorname{could}$ be covered in the maximal-mixing scenario while no sensitivity is possible in the MSSM in the scenarios where the mass of the lighter scalar is below $\sim 120 \mathrm{GeV} / c^{2}$.

\section{Associated production channels}

The isorate curves for the $\mathrm{h} \rightarrow \gamma \gamma$ channel in the associated production combining the $\mathrm{qq} \rightarrow \mathrm{Wh}$ and $\mathrm{q} \overline{\mathrm{q}} / \mathrm{gg} \rightarrow \mathrm{t} \overline{\mathrm{th}}$ processes are shown in Fig. 13 in the maximal-mixing scenario. The branching ratio for the $\mathrm{W} \rightarrow \ell \nu_{\ell}$ decay is included. The qq $\rightarrow \mathrm{Wh}$ process dominates the production and is large at $\operatorname{small} \tan \beta$, enhancing the total rate in this region. The cross section of the associated production is not sensitive to the mixing and stop mass effects. The production rate can be only affected through the loop mediated $\mathrm{h} \rightarrow \gamma \gamma$ decay process.

In the $\mathrm{SM}$, the $\mathrm{H} \rightarrow \gamma \gamma$ decay channel has been shown to be accessible in the associated qq $\rightarrow$ WH production process with an integrated luminosity of $100 \mathrm{fb}^{-1}$ [25]. The total production rate required for a $5 \sigma$ statistical significance in the $\mathrm{H} \rightarrow \gamma \gamma$ decay channel is between 0.8 and $0.6 \mathrm{fb}$ for $110<\mathrm{m}_{\mathrm{H}}<127 \mathrm{GeV} / c^{2}$. In the MSSM such a rate is expected only in the region of large $\mathrm{m}_{\mathrm{A}}$ and $\operatorname{small} \tan \beta$ as can be seen from Fig. 13. The $\mathrm{H} \rightarrow \mathrm{b} \overline{\mathrm{b}}$ decay channel has been investigated in the associated $\mathrm{q} \overline{\mathrm{q}} \rightarrow \mathrm{t} \overline{\mathrm{t}} \mathrm{H}$ process [26]. A $5 \sigma$ statistical significance is reached in the SM with integrated luminosities exceeding $40 \mathrm{fb}^{-1}$ around $\mathrm{m}_{\mathrm{H}} \sim 120 \mathrm{GeV} / c^{2}[26]$. Due to the enhanced $\mathrm{h} \rightarrow \mathrm{b} \overline{\mathrm{b}}$ couplings at large $\tan \beta$, a significant region has been shown to be covered with this decay channel in the MSSM [30].

\section{Weak gauge boson fusion production channels}

The SM Higgs boson is expected to be accessible in the weak gauge boson fusion production process qq $\rightarrow \mathrm{qqH}$ for $\mathrm{m}_{\mathrm{H}} \lesssim 150 \mathrm{GeV} / c^{2}$ with the $\mathrm{H} \rightarrow \gamma \gamma$ [31], $\mathrm{H} \rightarrow$ $\mathrm{WW}^{*} \rightarrow \ell^{+} \ell^{-} \nu_{\ell} \nu_{\ell}[32]$ and $\mathrm{H} \rightarrow \tau^{+} \tau^{-}$[27] decay channels for integrated luminosities exceeding $\sim 60 \mathrm{fb}^{-1}$. For the $\mathrm{H} \rightarrow \gamma \gamma$ decay channel the total rate required with 


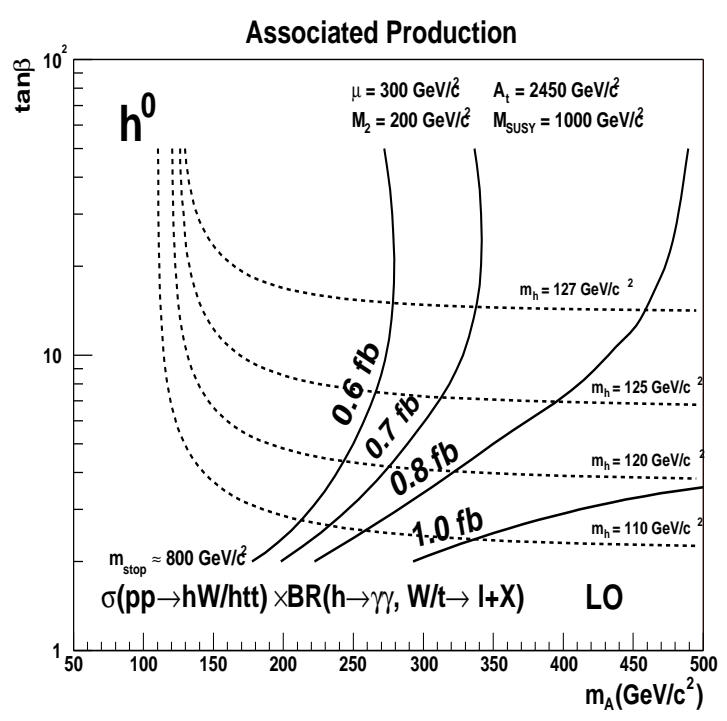

Figure 13. Isorate curves for $\mathrm{h} \rightarrow \gamma \gamma$ in the associated production processes qq $\rightarrow$ Wh and $\mathrm{q} \overline{\mathrm{q}} / \mathrm{gg} \rightarrow \mathrm{t} \overline{\mathrm{th}}$ with $\gamma \gamma \ell$ final states. Maximal stop mixing and LO cross sections assumed.

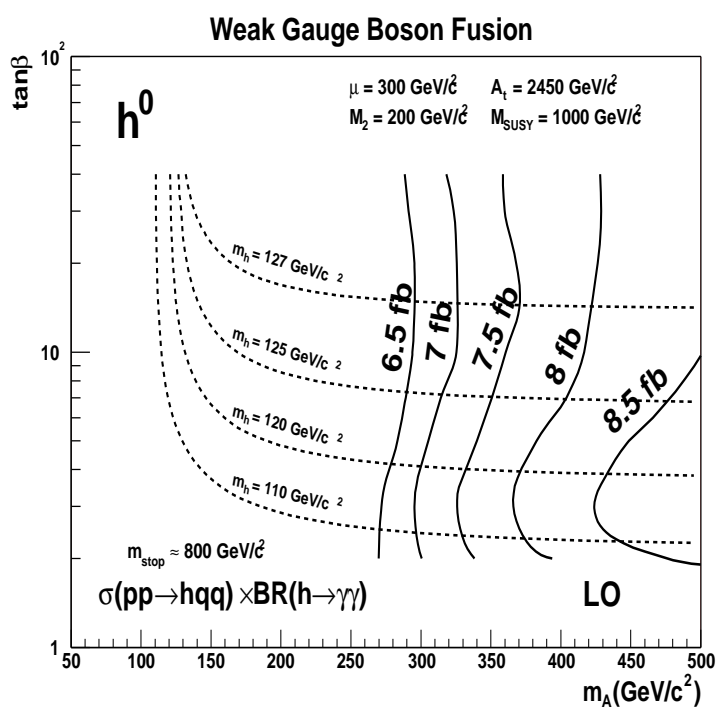

Figure 14. Isorate curves for weak gauge boson fusion $\mathrm{qq} \rightarrow \mathrm{qqh}, \mathrm{h} \rightarrow \gamma \gamma$ with maximal stop mixing.

$60 \mathrm{fb}^{-1}$ is about $8 \mathrm{fb}$ for $\mathrm{m}_{\mathrm{H}}=115 \mathrm{GeV} / \mathrm{c}^{2}$ and $6.6 \mathrm{fb}$ for $\mathrm{m}_{\mathrm{H}}=127 \mathrm{GeV} / \mathrm{c}^{2}$ [31]. The $\mathrm{H} \rightarrow \tau^{+} \tau^{-}$channel has been studied with lepton-plus-jet final states [27]. The total rate required for a $5 \sigma$ statistical significance with an integrated luminosity of $30 \mathrm{fb}^{-1}$ varies from 0.4 to $0.28 \mathrm{fb}$ for $115<\mathrm{m}_{\mathrm{H}}<127 \mathrm{GeV} / \mathrm{c}^{2}$ and from 0.28 to $0.19 \mathrm{fb}$ in the interval of $127<\mathrm{m}_{\mathrm{H}}<145 \mathrm{GeV} / c^{2}$ for the searches of the SM-like heavy scalar H. Figures 14 and 15 show the isorate curves for the $\mathrm{h} \rightarrow \gamma \gamma$ and $\mathrm{h} \rightarrow \tau^{+} \tau^{-}$decay channels in the weak gauge boson fusion process in the maximal-mixing scenario with LO cross sections. Figure 16 shows the corresponding isorate curves for the heavy scalar MSSM Higgs boson $\mathrm{H}$ in the $\mathrm{H} \rightarrow \tau^{+} \tau^{-}$decay channel. As can be seen seen from Fig. 15, the $\mathrm{h} \rightarrow \tau^{+} \tau^{-}$channel could be accessible in a large part of the parameter space already with low integrated luminosities. A sensitivity at large $\mathrm{m}_{\mathrm{A}}$ and $\tan \beta$ is expected also in the $\mathrm{h} \rightarrow \mathrm{WW}^{*} \rightarrow \ell^{+} \ell^{-} \nu_{\ell} \nu_{\ell}$ decay channel because the studies in the SM framework indicate a $5 \sigma$ discovery for $\mathrm{m}_{\mathrm{H}} \gtrsim 120 \mathrm{GeV} / c^{2}$ [32].

\section{Discovery potential}

Figures 17 and 18 show the discovery potential of CMS for the lightest MSSM Higgs boson as a function of $\mathrm{m}_{\mathrm{A}}$ and $\tan \beta$ assuming maximal stop mixing, $\mathrm{m}_{\text {top }}=175 \mathrm{GeV} / \mathrm{c}^{2}$ and $\mathrm{M}_{\mathrm{SUSY}}=1 \mathrm{TeV} / c^{2}$, for $30 \mathrm{fb}^{-1}$ and $100 \mathrm{fb}^{-1}$, respectively. The $\mathrm{h} \rightarrow \gamma \gamma$ and $\mathrm{h} \rightarrow \mathrm{ZZ}^{*} \rightarrow \ell^{+} \ell^{-} \ell^{\prime+} \ell^{\prime-}$ decay channels in the inclusive production are shown with NLO cross sections. With an integrated luminosity of $100 \mathrm{fb}^{-1}$ these channels cover a major part of the MSSM parameter space, for $\mathrm{m}_{\mathrm{A}} \gtrsim 200 \mathrm{GeV} / c^{2}$ and $\mathrm{m}_{\mathrm{A}} \gtrsim 250 \mathrm{GeV} / c^{2}$, 


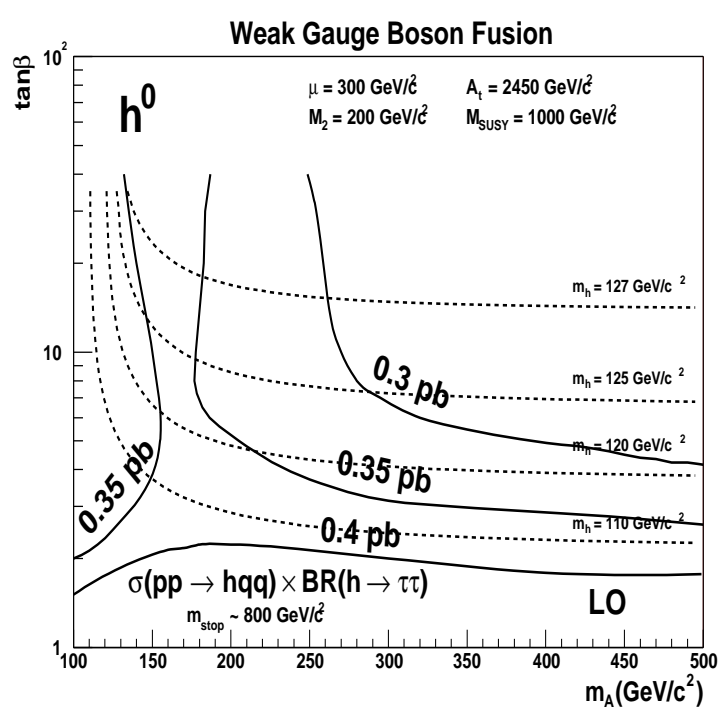

Figure 15. Isorate curves for $\mathrm{h} \rightarrow \tau^{+} \tau^{-}$in the weak gauge boson fusion qq $\rightarrow$ qqh. Maximal stop mixing and LO cross sections are assumed.

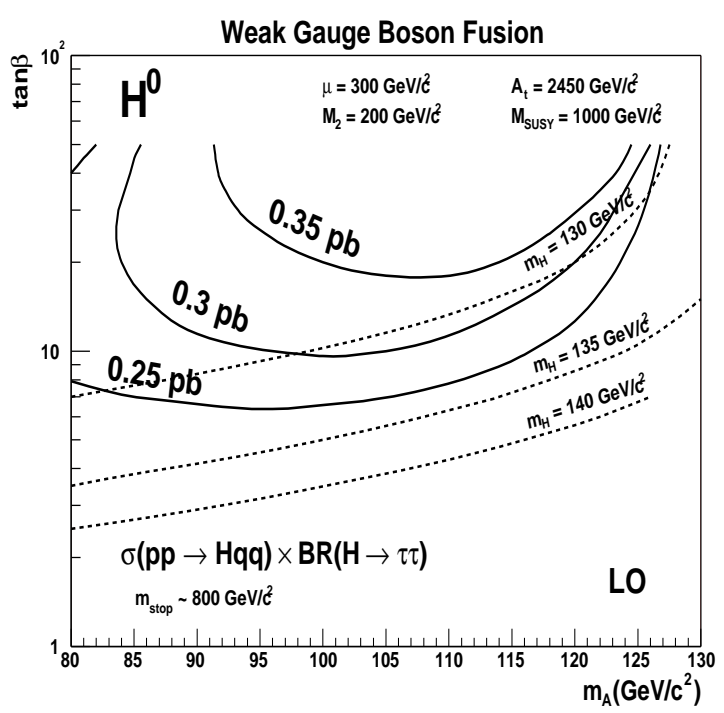

Figure 16. Isorate curves for $\mathrm{H} \rightarrow \tau^{+} \tau^{-}$in the weak gauge boson fusion qq $\rightarrow \mathrm{qqH}$ in the region of the $\left(\mathrm{m}_{\mathrm{A}}, \tan \beta\right)$ parameter space where $\mathrm{H}$ is SMlike. Maximal stop mixing and LO cross sections are assumed.

respectively. In the associated qq $\rightarrow$ Wh production the $\mathrm{h} \rightarrow \gamma \gamma$ channel covers only a small region at large $\mathrm{m}_{\mathrm{A}}$ and small $(\lesssim 5) \tan \beta$ values. The sensitivity for the $\mathrm{h} \rightarrow \mathrm{b} \overline{\mathrm{b}}$ channel in the associated $\mathrm{q} \overline{\mathrm{q}} / \mathrm{gg} \rightarrow \mathrm{t} \overline{\mathrm{t}} \mathrm{g}$ production with $60 \mathrm{fb}^{-1}$ from Ref. [30] is also shown in the figure. The reach in the $\mathrm{h} \rightarrow \gamma \gamma$ and $\mathrm{h} \rightarrow \tau^{+} \tau^{-}$decay channels in the weak gauge boson fusion production is shown in Fig. 17 for 60 and $30 \mathrm{fb}^{-1}$, respectively. In this production mode, the region $\tan \beta \gtrsim 5$ can be covered with the $\mathrm{h} \rightarrow \gamma \gamma$ channel for $\mathrm{m}_{\mathrm{A}} \gtrsim 350 \mathrm{GeV} / c^{2}$ and with the $\mathrm{h} \rightarrow \tau^{+} \tau^{-}$channel for $\mathrm{m}_{\mathrm{A}} \gtrsim 120 \mathrm{GeV} / \mathrm{c}^{2}$. The $\mathrm{H} \rightarrow \tau^{+} \tau^{-}$decay channel of the heavy scalar, shown also in Fig. 17, covers the region $\mathrm{m}_{\mathrm{A}} \lesssim 125 \mathrm{GeV} / \mathrm{c}^{2}$ in the weak gauge boson fusion. The region $90 \mathrm{GeV} / c^{2} \lesssim \mathrm{m}_{\mathrm{A}} \lesssim 130 \mathrm{GeV} / c^{2}$ at large $\tan \beta$, where the lightest Higgs boson is no more SM-like, is outside the reach of the channels discussed in this paper. To explore this region, the $\mathrm{h} \rightarrow \mu^{+} \mu^{-}$and $\mathrm{h} \rightarrow \tau^{+} \tau^{-}$decay channels can be used in the associated production with $\mathrm{b}$ quarks, $\mathrm{q} \overline{\mathrm{q}} / \mathrm{gg} \rightarrow \mathrm{b} \overline{\mathrm{b}} \mathrm{h}$, exploiting the enhanced couplings to down type fermions in the MSSM at large $\tan \beta$.

\section{Conclusions}

The production of the lightest MSSM Higgs boson h was studied, effects of SUSY parameters were discussed. The discovery potential was evaluated for CMS in the maximal-mixing scenario for the inclusive $\mathrm{h} \rightarrow \gamma \gamma$ channel, for the $\mathrm{h} \rightarrow \gamma \gamma$ channel in 


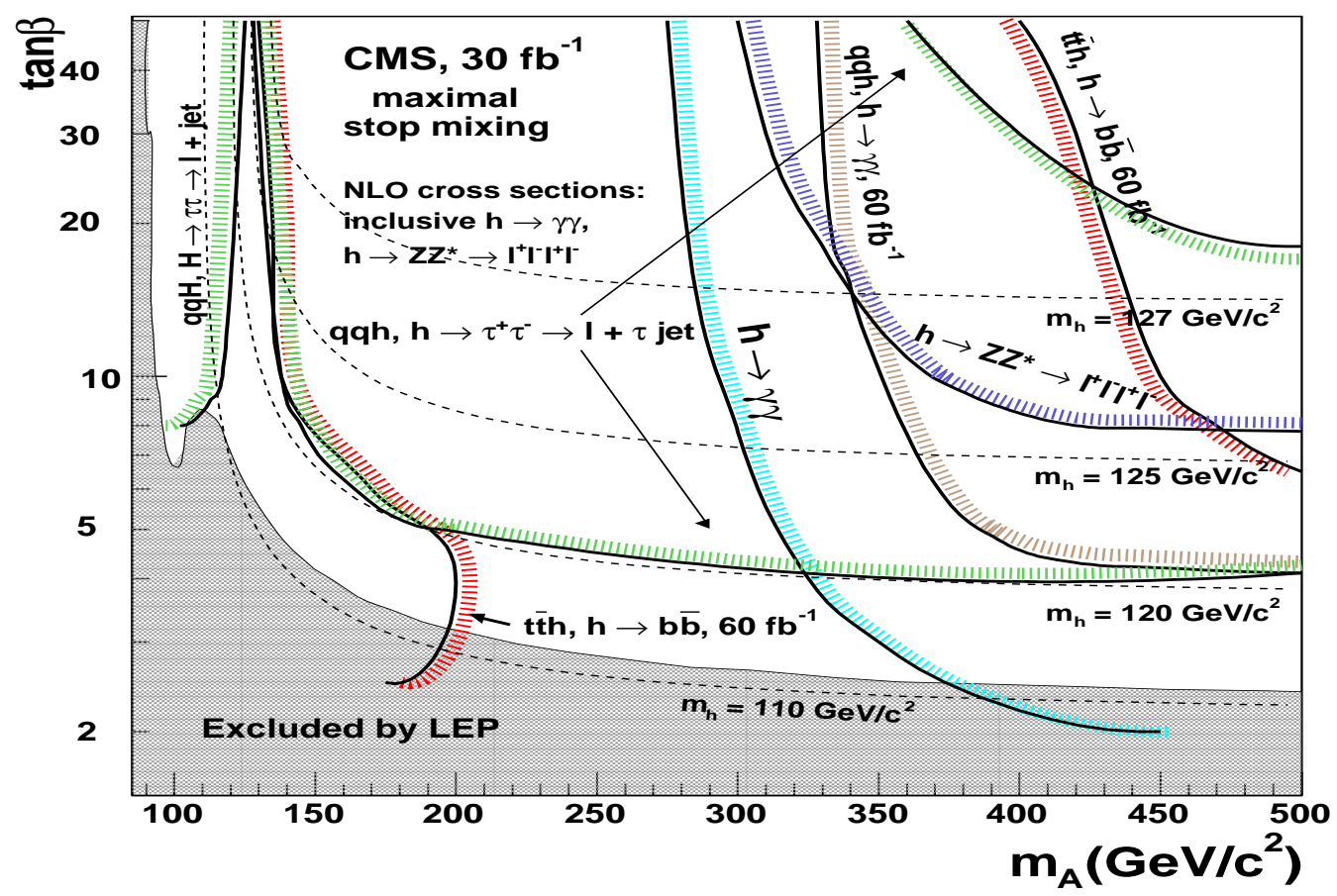

Figure 17. The $5 \sigma$-discovery potential of CMS for the lightest MSSM Higgs boson as a function of $\mathrm{m}_{\mathrm{A}}$ and $\tan \beta$ for $30 \mathrm{fb}^{-1}$ with maximal stop mixing. The reach in the

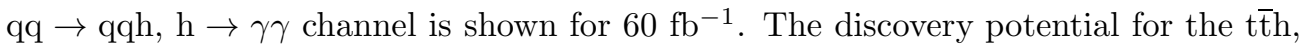
$\mathrm{h} \rightarrow \mathrm{b} \overline{\mathrm{b}}$ channel for $60 \mathrm{fb}^{-1}$ is taken from Ref. [30]. The reach of the $\mathrm{H} \rightarrow \tau^{+} \tau^{-}$decay channel of the heavy scalar in the $\mathrm{qq} \rightarrow \mathrm{qqH}$ production process is also shown.

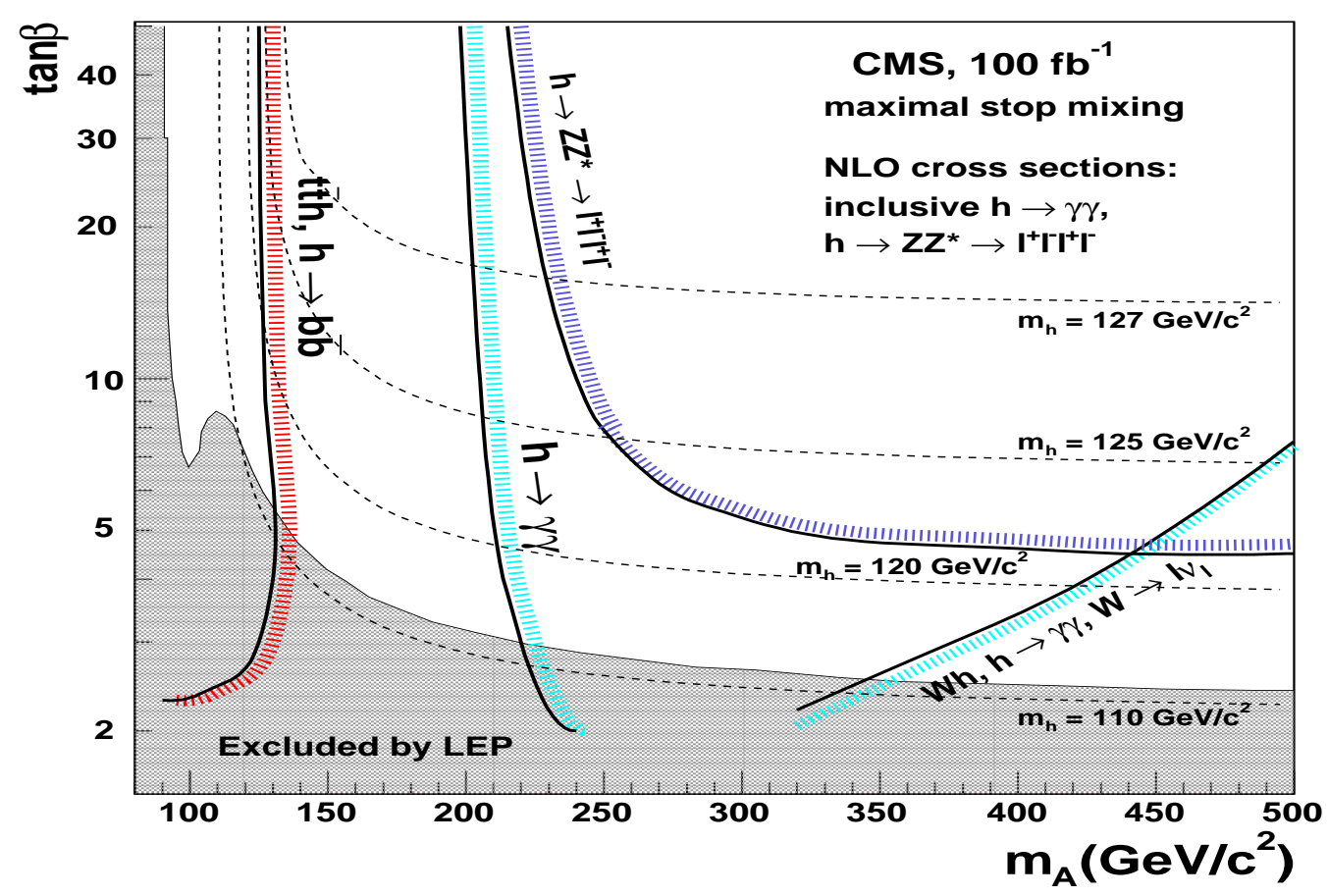

Figure 18. The $5 \sigma$-discovery potential for the lightest MSSM Higgs boson as a function of $\mathrm{m}_{\mathrm{A}}$ and $\tan \beta$ for $100 \mathrm{fb}^{-1}$ with maximal stop mixing. The discovery potential for the $t \bar{t} h, h \rightarrow b \bar{b}$ channel is taken from Ref. [30]. 
the associated production Wh and tthh, for the $\mathrm{h} \rightarrow \tau^{+} \tau^{-}$channel in the weak gauge boson fusion and, for the first time, for the $\mathrm{h} \rightarrow \mathrm{ZZ}^{*} \rightarrow \ell^{+} \ell^{-} \ell^{\prime+} \ell^{\prime-}$ channel at large $\tan \beta$. Consequencies of a light stop quark were shown for the expected discovery regions.

Already with an integrated luminosity of $30 \mathrm{fb}^{-1}$ the parameter space $\mathrm{m}_{\mathrm{A}} \gtrsim 150 \mathrm{GeV} / c^{2}$ and $\tan \beta \gtrsim 5$, apart from a small region at large $\mathrm{m}_{\mathrm{A}}$ and $\tan \beta$, is covered with the $\mathrm{h} \rightarrow \tau^{+} \tau^{-}$decay channel in the weak gauge boson fusion qq $\rightarrow$ qqh. The reach with the $\mathrm{h} \rightarrow \gamma \gamma$ decay channel with $30 \mathrm{fb}^{-1}$ is for $\mathrm{m}_{\mathrm{A}} \gtrsim 300 \mathrm{GeV} / c^{2}$ in the inclusive production and for $\mathrm{m}_{\mathrm{A}} \gtrsim 350 \mathrm{GeV} / c^{2}$ in the $\mathrm{qq} \rightarrow \mathrm{qqh}$ production process. With $60 \mathrm{fb}^{-1}$ the parameter space $150 \lesssim \mathrm{m}_{\mathrm{A}} \lesssim 400 \mathrm{GeV} / c^{2}\left(\mathrm{~m}_{\mathrm{A}} \gtrsim 150 \mathrm{GeV} / c^{2}\right.$ for $\tan \beta \lesssim 5)$ is covered with the $\mathrm{h} \rightarrow \mathrm{b} \overline{\mathrm{b}}$ decay channel in the $\mathrm{q} \overline{\mathrm{q}} / \mathrm{gg} \rightarrow \mathrm{t} \overline{\mathrm{t}} \mathrm{h}$ production process. With the large integrated luminosity of $100 \mathrm{fb}^{-1}$ the inclusive $\mathrm{h} \rightarrow \gamma \gamma$ channel yields a $5 \sigma$-discovery for $\mathrm{m}_{\mathrm{A}} \gtrsim 200 \mathrm{GeV} / c^{2}$ and the $\mathrm{h} \rightarrow \mathrm{ZZ}^{*} \rightarrow \ell^{+} \ell^{-} \ell^{\prime+} \ell^{\prime-}$ channel for $\mathrm{m}_{\mathrm{A}} \gtrsim 250 \mathrm{GeV} / \mathrm{c}^{2}, \tan \beta \gtrsim 5$.

The effects of loop corrections to the cross sections and branching ratios were studied in a scenario with large mixing and light stop quark. The consequences of the stop-top interference effects were shown for the $\mathrm{h} \rightarrow \gamma \gamma$ decay channel in the gluon fusion and in the inclusive production. The reduction of the total production rate was found to be significant for $\mathrm{m}_{\text {stop }} \lesssim 300 \mathrm{GeV} / c^{2}$. For $\mathrm{m}_{\text {stop }} \lesssim 200 \mathrm{GeV} / c^{2}$ the sensitivity in the inclusive $\mathrm{h} \rightarrow \gamma \gamma$ channel could be entirely lost. In this scenario the production rate is slightly enhanced for the associated production processes $\mathrm{q} \overline{\mathrm{q}} / \mathrm{gg} \rightarrow \mathrm{t} \overline{\mathrm{t}} \mathrm{h}$ and $\mathrm{qq} \rightarrow \mathrm{Wh}$ and in the weak gauge boson fusion qq $\rightarrow$ qqh process due to the positive interference effects on the $\mathrm{h} \rightarrow \gamma \gamma$ decay width.

\section{Acknowledgments}

The authors would like to thank Michael Spira for helpful comments and for his efforts in developing the program HIGLU compatible with the other programs used in this work. P.S. and S.L. would also like to thank Katri Huitu for helpful discussions.

[1] S. Heinemeyer, W. Hollik and G. Weiglein, Eur. Phys. Jour. C9 (1999) 343 (hep-ph/9812472); G. Degrassi, S. Heinemeyer, W. Hollik, P. Slavich and G. Weiglein, Eur. Phys. Jour. C28 (2003) 133 (hep-ph/0212020).

[2] LEP Higgs Working Group, Searches for the Neutral Higgs Bosons of the MSSM, hep-ex/0107030.

[3] Combination of the CDF and D0 results on the top quark mass, hep-ex/0404010.

[4] The LEP Higgs Working Group, http://lephiggs.web.cern.ch/LEPHIGGS/papers/ August2004_MSSM/LHWG-Note-2001-01.ps.

[5] A. Djouadi, Squark effects on Higgs boson production and decay at the LHC, Phys. Lett. B435 (1998) 101-108 (hep-ph/9806315) and references therein.

[6] DELPHI Collaboration, Searches for supersymmetric particles in $\mathrm{e}^{+} \mathrm{e}^{-}$collisions up to $208 \mathrm{GeV}$ and interpretation of the results within MSSM, CERN-EP/2003-007.

[7] LEP SUSY Work Group http://lepsusy.web.cern.ch/lepsusy/www/squarks_summer02/squark.ps.gz.

[8] R. Demina, J.D. Lykken, K. Matchev and A. Nomerotski, Phys. Rev. D62 (2000) 035011.

[9] M. Carena, S. Heynemeyer, C.E.M. Wagner and G. Weiglein, Suggestions for improved benchmark scenarios for Higgs-boson searches at LEP2, CERN-TH/99-374, DESY 99-186, hep-ph/9912223; M. Carena, S. Heynemeyer, C.E.M. Wagner and G. Weiglein, Suggestions for Benchmark 
Scenarios for MSSM Higgs Boson Searches at Hadron Colliders (hep-ph/0202167) and references therein.

[10] R. Kinnunen, S. Lehti, A. Nikitenko and S. Rantala, Effects of large mixing and light stop for $\mathrm{h} \rightarrow \gamma \gamma$ in MSSM, CMS Note 2000/043.

[11] T. Plehn, D.L. Rainwater and D. Zeppenfeld, A method identifying $\mathrm{H} \rightarrow$ tau + tau $\rightarrow \mathrm{e}+\mathrm{mu}+\mathrm{p}(\mathrm{T})$ at the CERN LHC, FERMILAB-PUB-99-290-T, 1999.

[12] S. Abdullin et al., Summary of the CMS Potential for the Higgs Boson Discovery, CMS NOTE$2003 / 033$.

[13] M. Spira, HIGLU, hep-ph/9510347.

[14] A.Djouadi, J. Kalinowski and M. Spira, HDECAY: a Program for Higgs Boson Decays in the Standard Model and its Supersymmetric Extension, Comput. Phys. Commun. 108 (1998) 56.

[15] M. Spira, http://mspira.home.cern.ch/mspira/proglist.html

[16] M.Spira et. al, Nucl.Phys. B 453 (1995) 17-82.

[17] S. Dawson et. al., hep-ph/9603423 "QCD Corrections to SUSY Higgs Production: The Role of Squark Loops".

[18] J. Ellis and D. Rudaz, Phys. Lett. B128 (1983) 248.

[19] A. Djouadi et. al., The coupling of the lightest SUSY Higgs on two photons in the decoupling regime, Eur. Phys. J. C 1, 149-162 (1998).

[20] R. Harlander, Supersymmetric Higgs Production at the Large Hadron Collider, hep-ph/0311005.

[21] L3 Collaboration, Phys. Lett. B580 (2004) 37-49.

[22] CMS Collaboration, The Electromagnetic Calorimeter Project, Technical Design Report, CERN/LHCC 97-33, CMS TDR 4, 1997.

[23] CMS Collaboration, The Tracker Project, Technical Design Report, CERN/LHCC 98-6, CMS TDR 5, 1998.

[24] CMS Collaboration, The Muon Project, Technical Design Report, CERN/LHCC 97-32, CMS TDR 3, 1997.

[25] M.N. Dubinin, V.A. Ilyin and V.I. Savrin, Light Higgs Boson Signal at LHC in the Reactions pp $\rightarrow \gamma \gamma+$ jet and pp $\rightarrow \gamma \gamma+$ lepton, CMS NOTE 1997/101; S. Abdullin et al., Phys. Lett., B431 (1998) 410.

[26] V. Drollinger, Th. Muller and D. Denegri, Searching for Higgs Bosons in Association with Top Quark Pairs in the $\mathrm{H} \rightarrow$ b $\bar{b}$ Decay Mode, CMS NOTE 2001/054, hep-ph/0111312; V. Drollinger, Th. Muller and D. Denegri, Prospects for Higgs Bosons Searches in the Channel $\mathrm{W}^{ \pm} \mathrm{H} \rightarrow \ell \mathrm{b} \overline{\mathrm{b}}$, CMS NOTE 2002/006, hep-ph/0201249.

[27] G. Azuelos et al., Search for the SM Higgs boson using vector boson fusion at the LHC, Workshop on Physics at TeV Colliders, Les Houches, France, 2001, hep-ph/0203056.

[28] I. Iashvili, R. Kinnunen, A. Nikitenko and D. Denegri, Study of the H $\rightarrow Z^{*} \rightarrow \ell^{+} \ell^{-} \ell^{\prime+} \ell^{\prime-}$ Channel in CMS, CMS TN/95-059.

[29] K. Lassila-Perini, Dissertation, ETH No. 12961 (1998).

[30] V. Drollinger, Private communication.

[31] M. Dubinin, Higgs Boson Signal in the Reaction pp $\rightarrow \gamma \gamma+$ two Forward Jets, CMS NOTE $2001 / 022$.

[32] N. Akchurin et al., Study of Low Mass Higgs Using pp $\rightarrow$ qqH at CMS, CMS NOTE-2002/016. 